\title{
Optimal Selection and Ordering of Columns in Supersaturated Designs
}

\author{
N. Niki ${ }^{a}$ \\ a Tokyo University of Science, Department of Management Science, \\ Kagurazaka, Shinjuku-ku, Tokyo 162-8601, Japan. \\ M. Iwata ${ }^{b}$ \\ ${ }^{\mathrm{b}}$ DENSO Corporation, \\ Showa-cho, Kariya, Aichi 448-8661, Japan. \\ H. Hashiguchi ${ }^{\mathrm{c}, *}$ \\ 'Saitama University, Graduate School of Science and Engeering, \\ Shimo-Okubo, Sakura, Saitama 338-8570, Japan. \\ S. Yamada ${ }^{\mathrm{d}}$ \\ ${ }^{\mathrm{d}}$ University of Tsukuba, Graduate School of Business Sciences, \\ Otsuka, Bunkyo-ku, Tokyo 112-0012, Japan.
}

\begin{abstract}
Two methods to select columns for assigning factors to work on supersaturated designs are proposed. The focus of interest is the degree of non-orthogonality between the selected columns. One method is the exhaustive enumeration of selections of $p$ columns from all $k$ columns to find the exact optimality, while the other is intended to find an approximate solution by applying techniques used in the corresponding analysis, aiming for ease of use as well as a reduction in the large computing time required for large $k$ with the first method. Numerical illustrations for several typical design matrices reveal that the resulting "approximately" optimal assignments of factors to their columns are exactly optimal for any $p$. Ordering the columns in $\mathrm{E}\left(s^{2}\right)$-optimal designs results in promising new findings including a large number of $\mathrm{E}\left(s^{2}\right)$-optimal designs.
\end{abstract}

Key words: corresponding analysis, exhaustive enumeration, factor assignment, non-orthogonality, optimal design 


\section{Introduction}

Supersaturated designs are, essentially, fractional factorial designs of which can be assigned more factors than the standard fractional factorial design. They are helpful for screening many factors to find active factors from many candidates such as the primary stage of a scientific investigation, technology development and product innovation. The designs are utilized under assumption of the effect sparsity, i.e. a small number of factors are active while the remaining factors are not. A distinctive aspect of supersaturated designs is that the degree of non-orthogonality between two columns, e.g., the inner products of the two columns in two-level designs, does not always vanish to zero. Following pioneering studies by Satterthwaite (1959) and Booth and Cox (1962), Lin (1993) has introduced a method using half fractions of Hadamard matrix to construct supersaturated designs. This simple and practical method has inspired a number of active researchers in the field of experimental design.

The first aim to construct supersaturated designs is to attempt to minimize the degree of non-orthogonality under given numbers of runs and columns. One promising approach to find a solution to this requirement is to construct a design any two of columns of which have a lower measure of non-orthogonality at least on an average. In fact, many researchers have proposed designs with optimality in $\mathrm{E}\left(s^{2}\right)$, which is an average of the squared inner products between all paired columns. Most of the previous studies including Wu (1993), Lin (1995), Nguyen (1996), Cheng (1997), Li and Wu (1997), Tang and Wu (1997), Yamada and Lin (1997), Butler et al. (2001), Lejeune (2003), Bulutoglu and Cheng (2004), and Nguyen and Cheng (2008) have been devoted to the construction methodologies for two-level supersaturated designs with better orthogonality in term of $\mathrm{E}\left(s^{2}\right)$. For other types of supersaturated designs, Yamada and Lin (1999) and Yamada et al. (1999) have introduced a class of three-level supersaturated designs, then, which has been extended to mixed-level by Yamada and Matsui (2002) and Yamada and Lin (2002). Some construction methods for optimal mixed-level supersaturated designs have been discussed in Chen and Liu (2008) and Nguyen and Liu (2008) as a recent work.

In many practical cases, the technical knowledge in the experimented field implies that the possibilities of active factor are not uniform over the candidate factors. One typical case is that some of the candidate factors are assumed to be more important than the remaining factors. In order to utilize the technical knowledge, the factor with high possibility of activeness should be assigned the columns whose degree of non-orthogonality with the other columns are low,

\footnotetext{
* Corresponding Author

Email address: hiro@mail.saitama-u.ac.jp (H. Hashiguchi).
} 
while the remaining factors are assigned the remaining columns whose degree of non-orthogonality with the other columns are not low. Since the previous studies have attempted to minimize the average of degree of non-orthogonality over all paired columns, it is not possible to assign factors to columns with considering the possibility of activeness.

In order to solve this problem, this paper discusses the optimality for ordering of columns in a supersaturated design, where this ordering is to arrange columns in the left hand side and dependent columns in the right hand side of a supersaturated design. The ordered designs have a property that the degree of non-orthogonality is low by selecting the columns from the left hand side to any column. This property assures that the degree of non-orthogonality is low for the important factors by assigning factors from the left column based on the possibility.

Section 2, discusses two approaches to optimal selection of the columns from an existing design. One is the exhaustive enumeration of selections of $p$ columns from $k$ columns to obtain the exact optimality, while the other is intended to find an approximate solution by ordinating the columns of a design matrix, as in corresponding analysis, for ease of use as well as to avoid a combinatoric explosion in computation.

Numerical illustrations are provided in Section 3 to demonstrate the tactics required in ordination and additional discussion concerning the optimal designs including all $8 \times k \mathrm{E}\left(s^{2}\right)$-optimal designs for $k \leq 35$ aligned in only one design matrix with ordinated columns. This example shows that an arrangement of 35 columns realize an overall optimal design, namely, from the 18th to 35th column leads to overall optimal and that, by reverse arrangement, from the 4 th to 17 th column does so as well.

\section{Selection of columns}

\subsection{Definitions and preliminaries}

Let $D$ be the $n \times k$ design matrix of a $t$-level supersaturated design with $n$ runs and $k$ columns, i.e.,

$$
\begin{array}{r}
D=\left(d_{i j}\right)=\left(\boldsymbol{d}_{1} \ldots \boldsymbol{d}_{k}\right), \quad d_{i j} \in T=\{1,2 \ldots, t\} \\
i \in N=\{1,2 \ldots, n\}, \quad j \in K=\{1,2 \ldots, k\} .
\end{array}
$$


where $n-1<k(t-1)$. We treat a balanced design with equal occurrence of $\{1,2, \ldots t\}$ in $d_{i, j}$.

Then the $\chi^{2}$ value is defined as

$$
\chi_{i j}^{2}=\sum_{a=1}^{t} \sum_{b=1}^{t} \frac{\left(\#\left\{r \in N \mid d_{r i}=a, d_{r j}=b\right\}-n / t^{2}\right)^{2}}{n / t^{2}}
$$

which is proportional to the measure proposed by Booth and Cox (1962) in the case of $t=2$. When $t=2$, i.e., for two-level designs, the measures

$$
s_{i j}^{2}=\left(\left(2 \boldsymbol{d}_{i}-3 \mathbf{1}_{n}\right)^{\top}\left(2 \boldsymbol{d}_{j}-3 \mathbf{1}_{n}\right)\right)^{2}=n \chi_{i j}^{2}
$$

and

$$
\mathrm{E}\left(s^{2}\right)=\left(\begin{array}{l}
k \\
2
\end{array}\right)^{-1} \sum_{1 \leq i<j \leq k} s_{i j}^{2}=n \chi^{2}(K),
$$

are commonly used for representing non-orthogonality between the two and all the $k$ columns, respectively, where $\mathbf{1}_{n}$ is the $n$-dimensional column vector of all ones. Hereafter, for convenience, $U=\left(u_{i j}\right)$ denotes the $k \times k$ matrix of $\chi^{2}$ values of which all the diagonal elements are zeroes provided as

$$
U=\left(\chi_{i j}^{2}\right)-\operatorname{diag}\left(\chi_{11}^{2}, \ldots, \chi_{k k}^{2}\right)
$$

It is clear that $U^{\top}=U$, where the superscript ${ }^{\top}$ denotes transposition.

Several papers including Yamada and Lin (1999) use the criterion

$$
\chi^{2}(K)=\left(\begin{array}{c}
k \\
2
\end{array}\right)^{-1} \sum_{1 \leq i<j \leq k} \chi_{i j}^{2}=\frac{1}{k(k-1)} \mathbf{1}_{k}^{\top} U \mathbf{1}_{k}
$$

to evaluate total non-orthogonality among columns of $D$, where $K$ is defined in (1)

For any $S=\left\{s_{1}, s_{2}, \ldots, s_{p}\right\} \in \mathcal{K}(p)$, where

$$
\mathcal{K}(p)=\left\{S \in 2^{K} \mid \# S=p \leq k\right\}
$$


let $D_{S}$ and $U_{S}$ denote the $n \times p$ submatrix $\left(\boldsymbol{d}_{s_{1}} \cdots \boldsymbol{d}_{s_{p}}\right)$ of $D$ and the $p \times p$ submatrix $\left(u_{s_{i} s_{j}}\right)$ of $U$, respectively. Then, in the same way as (6), we define the measure of non-orthogonality for the selected columns $D_{S}$ from $D$ as

$$
\chi^{2}(S)=\left(\begin{array}{c}
p \\
2
\end{array}\right)^{-1} \sum_{1 \leq i<j \leq p} \chi_{s_{i} s_{j}}^{2}=\frac{1}{p(p-1)} \mathbf{1}_{p}^{\top} U_{S} \mathbf{1}_{p}
$$

The set

$$
\mathcal{K}^{*}(p)=\left\{S \in \mathcal{K}(p) \mid{ }^{\forall} R \in \mathcal{K}(p), \chi^{2}(R) \geq \chi^{2}(S)\right\}
$$

is hereafter called the "optimal set". In other words, $\chi^{2}(S)$ attains its minimum value for any $S \in \mathcal{K}^{*}(p)$.

\subsection{Combinatoric method for exact optimality}

The following algorithm lexicographically finds the most precedent element $S^{*}$ in the optimal set $\mathcal{K}^{*}(p)$ by performing an exhaustive enumeration of all the $\left(\begin{array}{l}k \\ p\end{array}\right)$ elements of $\mathcal{K}(p)$ :

\section{Algorithm 1 (Lexicographical search for $S^{*}$ ).}

Input: $\quad k, p, U$.

Output: $S^{*}, m^{*}=\chi^{2}\left(S^{*}\right)$.

1: (Initialize) Initiate the search with $S^{*} \leftarrow\{1, \ldots, p\}, m^{*} \leftarrow \chi^{2}\left(S^{*}\right)$, $S \leftarrow S^{*}, a \leftarrow 0$, and $b \leftarrow 0$.

2: (Make the next selection in lexicographical order) If $b<k-a$ then set $b \leftarrow s_{p}, a \leftarrow 1$ and increment $s_{p} \leftarrow s_{p}+1$. Otherwise, $b \leftarrow s_{p-a}, a \leftarrow a+1$ and replace $s_{p-a+i} \leftarrow b+i$ for each $i=1, \ldots, a$.

3: (Check for optimality of $S$ ) Compute $m \leftarrow \chi^{2}(S)$. If $m<m^{*}$ then replace $S^{*} \leftarrow S$ and $m^{*} \leftarrow m$.

4: (Check for termination) If $s_{1}=k-p+1$ then return $m$ and $S^{*}$ to exit. Otherwise, go back to Step 2.

See Nijenhuis and Wilf (1978), for other methods and topics related to the enumeration of $\mathcal{K}(p)$.

Each supersaturated design would be distributed for public use with a list of the representative members of $\mathcal{K}^{*}(p)$ for each $p \in K$, obtained by computing $\chi^{2}(S)$ in $2^{k}-1$ times. 
If no list of optimal selections is provided, one would have to find one of the members of $\mathcal{K}^{*}(p)$ for provided $p$, when necessary, which may be tedious as well as highly time consuming. Typically, for large $k$ with $p$ close to $k / 2$, the required number $\left(\begin{array}{l}k \\ p\end{array}\right)$ of evaluations of the $\chi^{2}$ values is roughly evaluated as

$$
\left(\begin{array}{c}
k \\
p
\end{array}\right) \approx\left(\begin{array}{c}
k \\
k / 2
\end{array}\right) \approx \sqrt{\frac{2}{\pi}} \frac{2^{k}}{\sqrt{k}}
$$

which is sometimes too large to process even when using high-end computers.

\subsection{Ordering of columns for approximate optimality}

Consider a permutation $Q=\left(q_{1}, \ldots, q_{k}\right)$ of $K$ and its action upon the positions of columns in D, i.e.,

$$
D^{Q}=\left(\boldsymbol{d}_{q_{1}}, \ldots, \boldsymbol{d}_{q_{k}}\right)
$$

which provides a naturally ordered sequence of the selections:

$$
S_{1}=\left\{q_{1}\right\} \subset S_{2}=\left\{q_{1}, q_{2}\right\} \subset \cdots \subset S_{k}=\left\{q_{1}, \ldots, q_{k}\right\} .
$$

There may be several ways to define the "overall" non-orthogonality of

$$
D_{S_{1}}, D_{S_{2}}, \ldots, D_{S_{k}}
$$

or overall badness of the sequence of the top-leftmost submatrices of $U^{Q}$

$$
U_{S_{1}}, U_{S_{2}}, \ldots, U_{S_{k}}
$$

A permutation or ordering $Q$ is called "optimal", if it minimizes the overall non-orthogonality; and an optimal ordering $Q$ is called "strictly optimal", if $S_{p} \in \mathcal{K}^{*}(p)$ for any $p \in K$.

Use of $D^{Q}$ in place of $D$ with an optimal $Q$, preferably one that is strictly optimal, may make it unnecessary to provided a list for selection with $D$ or to find an optimum selection on the fly.

However, although optimal permutation in view of any well-defined overall badness can be obtained by enumerating all the $k$ ! permutations of $K$, the 


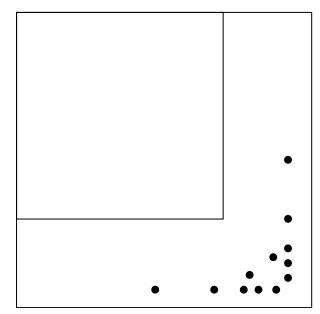

(a) Too idealistic

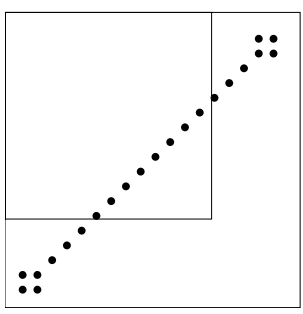

(b) Typically observed

Fig. 1. Distribution of large elements in optimally permuted $U$.

involved computation increases in size much faster than the $2^{k}$ required in the previous subsection.

One might consider that the optimal permutation $Q$ tends to provide a configuration of rows and columns to $U^{Q}$ as shown in (a) of Figure 1, where the dots represent the relatively large $\chi^{2}$ values in $U^{Q}$. On the contrary, the large values have been typically distributed as shown in (b) of Figure 1, in all the cases the authors have examined, since various types of symmetry are involved in any existing experimental design. The idea discussed here is to find an approximately optimum permutation that provides a shape resembling that in (b) through simultaneous ordering of rows and columns in $U$.

Let $\boldsymbol{x}=\left(x_{1}, x_{2}, \ldots, x_{k}\right)^{\top} \in \mathbb{R}^{k}(i \in K)$ be a vector of coordinates provided to the rows and columns of $U$ where the matrix $U$ is a set of $k^{2}$ observations

$$
\left\{\left(x_{i}, x_{j}, u_{i j}\right) \mid i, j \in K\right\}
$$

for two random variates $X, Y$ and the weight to $\left(x_{i}, x_{j}\right)$, respectively. Then, the correlation coefficient $\rho$ between $X$ and $Y$ may be used as a criterion for likeness to the shape in Figure 1 (b) if $\rho<0$.

Provided

$$
\bar{X}=\bar{Y}=\frac{1}{\ell} \mathbf{1}_{k}^{\top} F \boldsymbol{x}=0
$$

it holds that

$$
\sigma_{X}^{2}=\sigma_{Y}^{2}=\frac{1}{\ell} \boldsymbol{x}^{\top} F \boldsymbol{x}, \quad \sigma_{X Y}=\frac{1}{\ell} \boldsymbol{x}^{\top} U \boldsymbol{x}
$$

where

$$
F=\operatorname{diag}\left(U \mathbf{1}_{k}\right), \quad \ell=\mathbf{1}_{k}^{\top} F=k(k-1) \chi^{2}(K) .
$$


The necessary condition for $\rho$ to attain its minimum value at $\boldsymbol{x}$, subject to $\sigma_{X}^{2}=\sigma_{Y}^{2}=1$, is given by the Lagrange multiplier $\lambda$ as

$$
\frac{\partial}{\partial x_{i}}\left[\frac{1}{\ell} \boldsymbol{x}^{\top} U \boldsymbol{x}-\lambda\left(\frac{1}{\ell} \boldsymbol{x}^{\top} F \boldsymbol{x}-1\right)\right]=0 \quad(i \in K)
$$

which yields the eigenvalue problem

$$
U \boldsymbol{x}=\lambda F \boldsymbol{x},
$$

or, by letting $F=H^{2}, \boldsymbol{y}=H \boldsymbol{x}$ and $W=H^{-1} U H^{-1}$,

$$
W \boldsymbol{y}=\lambda \boldsymbol{y} .
$$

The eigenvector $\boldsymbol{x}_{*}$ associated with the smallest negative eigenvalue $\lambda_{*}$ is the solution required for approximately optimum ordering, since it holds from (20) that

$$
\rho=\frac{1}{\ell} \boldsymbol{x}^{\top} U \boldsymbol{x}=\frac{\lambda}{\ell} \boldsymbol{x}^{\top} F \boldsymbol{x}=\lambda .
$$

The ascending order of the elements in $\boldsymbol{x}_{*}$ yields the permutation $Q$ with which the columns of $D$ are rearranged.

Remark 1. The equations (20) and (21) have the largest eigenvalue 1 and its associated eigenvector $\mathbf{1}_{k}$ and $H \mathbf{1}_{k}$, respectively, from the definition of $F$ in (18). Since $W$ is symmetric, it holds for any eigenvector $\boldsymbol{x} \neq \mathbf{1}_{k}$ that

$$
\bar{X}=\frac{1}{\ell} \mathbf{1}_{k}^{\top} F \boldsymbol{x}=\frac{1}{\ell}\left(H \mathbf{1}_{k}\right)^{\top}(H \boldsymbol{x})=0,
$$

which means that the condition (16) is always satisfied.

Remark 2. For almost all the proposed supersaturated designs in the literature, including those in the bibliography of this article, there exists a positive constant $f$ such that $F=f I$, where $I$ is the identity matrix.

Remark 3. The characteristic equation $|W-\lambda I|=0$ in $\lambda$ has multiple roots, owing to the multiple symmetry incorporated in $D$. If the smallest root $\lambda_{*}$ is not simple, typically being a double root, the associated eigenvector $\boldsymbol{x}_{*}$ is not unique.

Any vector in the eigenspace is equivalent, but, in view of the usage, it may be preferable to place the elements of $\boldsymbol{x}_{*}$ so that they are spaced apart evenly. 
Remark 4. For solving the smallest eigenvalue problem (21) for a $k \times k$ symmetric matrix $W$, the computational cost requires only $O\left(k^{3}\right)$ arithmetic operations.

\subsection{Heuristic refinement}

Since the resulting permutation $Q$ provides only approximate optimality, although it is strictly optimal for many existing supersaturated designs, several types of refinement may be applicable.

The method that may be considered should be most effective for better $Q$ is to generate all the permutations of $K$ that differ from $Q$ only in the last few elements. It certainly works often but its drawback is the amount of computations of $O\left(k^{2} j !\right)$ required for replacing the last $j$ elements with the better ones.

A simpler and more promising refinement can be summarized in the algorithm shown below, which is based on the most probable fact that the first $\alpha$ elements of $Q$ provide the optimal selection, or at least close to optimal for a positive integer $\alpha$. The empirically-derived suitable value of $\alpha$ is slightly greater than $k / 2$ when $k$ is a few dozen or less. However, it seems preferable to reduce the ratio $\alpha / k$ as $k$ increases. In any case, a certain amount of trial and error would be necessary before the desired effect is achieved.

\section{Algorithm 2 (A promising refinement).}

Input: $\quad k, \alpha, Q=\left(q_{1}, \ldots, q_{k}\right), U$.

Output: $Q^{*}$.

(1) (Preparation 1) Divide $Q$ into the possibly optimal selection $L$ and the remaining part $R$, by letting $L \leftarrow\left\{q_{1}, \ldots, q_{\alpha}\right\}$ and $R \leftarrow\left\{q_{\alpha+1}, \ldots, q_{k}\right\}$. Set $Q^{*} \leftarrow()$, the empty vector.

(2) (Refinement of the right-hand part) Find $q^{*} \leftarrow \arg \min _{q \in R} \chi^{2}(L \cup\{q\})$, then update $L \leftarrow L \cup\left\{q^{*}\right\}, R \leftarrow R \backslash\left\{q^{*}\right\}$ and append $q^{*}$ to $Q^{*} \leftarrow\left(Q^{*} q^{*}\right)$. If several $q$ 's in $R$ provide the minimum $\chi^{2}$, the left-most element with the smallest index should be $q^{*}$. Repeat this step unless $R=\phi$.

(3) (Preparation 2) Reset $L \leftarrow\left\{q_{1}, \ldots, q_{\alpha}\right\}$ again.

(4) (Refinement of the left-hand part) Find $q^{*} \leftarrow \arg \min _{q \in L} \chi^{2}(L \backslash\{q\})$, then update $L \leftarrow L \backslash\left\{q^{*}\right\}$ and prepend $q^{*}$ to $Q^{*} \leftarrow\left(q^{*} Q^{*}\right)$. If several $q^{\text {'s in } L}$ provide the minimum $\chi^{2}$, the right-most element with the largest index should be $q^{*}$. Repeat this step unless $L=\phi$. 
Table 1

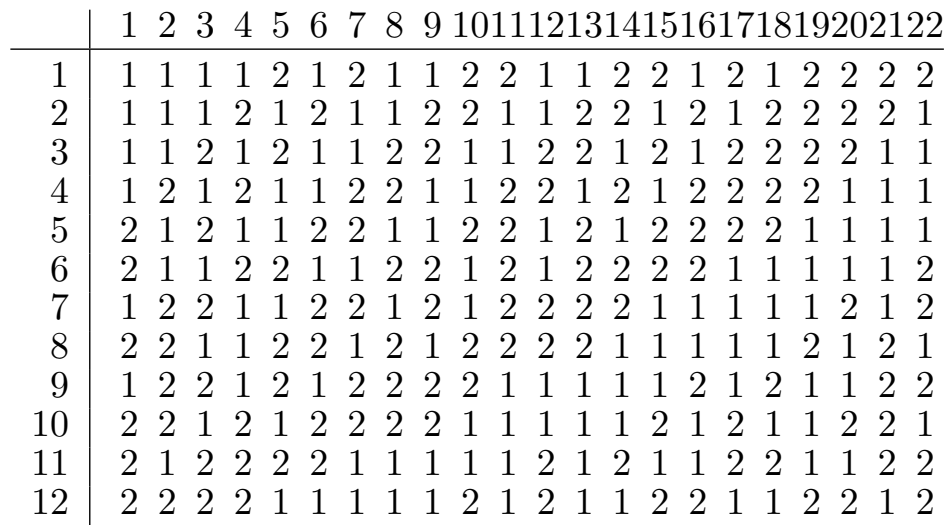

A supersaturated design $(t=2, n=12, k=22)$, Lin (1993).

Steps (3) and (4) may be unnecessary for practical use, since another design, typically an orthogonal one, would be applicable for $p \leq \alpha$. However, even in that case, this additional refinement should still be preferable for the sake of integrity.

With $Q$ or its refined version $Q^{*}$, each supersaturated design $D$ should be permuted as $D^{Q}$ or $D^{Q^{*}}$ before publication, without a long list of prioritybased selections.

\section{$3 \quad$ Numerical illustration}

\subsection{Ordering columns with additional refinement}

Table 1 shows a supersaturated design proposed by Lin (1993) for $t=2$, $n=12$ and $k=22$ and which is written as $D$ here. The matrix $U$ of $\chi^{2}$-values for this design is shown in Table 2 in which all the non-zero elements are $4 / 3$.

The results of exhaustive enumeration of $\mathcal{K}(p)$ for each $p$ from 22 down to 2 are summarized in Tables 3 and 4 , where $S^{*}$ denotes the most precedent element of $\mathcal{K}^{*}(p)$ in lexicographic order. For larger-scale designs, e.g., with $k>40$, it is almost impossible to find an exactly optimal selection of columns for each $p \in K$.

It is worth noting that,

(1) Although the number $\# \mathcal{K}(p)=\left(\begin{array}{l}k \\ p\end{array}\right)$ of straightforward selections rapidly increases as $p$ decreases toward $k / 2$, the number $\# \mathcal{K}^{*}(p)$ of optimal selections remains small.

(2) In cases where $p$ is close to $k / 2$, the probability $\# \mathcal{K}^{*}(p) / \# \mathcal{K}(p)$ that a member of $\mathcal{K}^{*}(p)$ will appear in random sampling $p$ from $k$ without re- 
Table 2

\begin{tabular}{r|llllllllllllllllllllll} 
& 1 & 2 & 3 & 4 & 5 & 6 & 7 & 8 & 9 & 10111 & 1314 & 151617181920 & 2122 \\
\hline 1 & 0 & 0 & 0 & 4 & 0 & 4 & 4 & 0 & 4 & 0 & 0 & 0 & 0 & 4 & 4 & 0 & 0 & 4 & 4 & 4 & 0 & 0 \\
2 & 0 & 0 & 0 & 0 & 4 & 0 & 4 & 4 & 0 & 0 & 0 & 4 & 4 & 4 & 4 & 0 & 4 & 4 & 0 & 0 & 0 & 0 \\
3 & 0 & 0 & 0 & 4 & 0 & 0 & 0 & 4 & 0 & 0 & 4 & 4 & 0 & 4 & 0 & 0 & 0 & 4 & 4 & 0 & 4 & 4 \\
4 & 4 & 0 & 4 & 0 & 4 & 0 & 4 & 0 & 0 & 4 & 4 & 0 & 4 & 4 & 0 & 4 & 0 & 0 & 0 & 0 & 0 & 0 \\
5 & 0 & 4 & 0 & 4 & 0 & 4 & 4 & 4 & 0 & 0 & 0 & 0 & 0 & 0 & 0 & 4 & 0 & 0 & 0 & 4 & 4 & 4 \\
6 & 4 & 0 & 0 & 0 & 4 & 0 & 0 & 4 & 0 & 0 & 0 & 0 & 4 & 0 & 4 & 4 & 0 & 0 & 4 & 0 & 4 & 4 \\
7 & 4 & 4 & 0 & 4 & 4 & 0 & 0 & 0 & 0 & 0 & 4 & 4 & 4 & 0 & 0 & 0 & 4 & 0 & 4 & 0 & 0 & 0 \\
8 & 0 & 4 & 4 & 0 & 4 & 4 & 0 & 0 & 4 & 4 & 0 & 0 & 0 & 4 & 0 & 0 & 0 & 0 & 0 & 4 & 0 & 4 \\
9 & 4 & 0 & 0 & 0 & 0 & 0 & 0 & 4 & 0 & 4 & 4 & 4 & 4 & 0 & 0 & 0 & 4 & 0 & 4 & 4 & 0 & 0 \\
10 & 0 & 0 & 0 & 4 & 0 & 0 & 0 & 4 & 4 & 0 & 0 & 4 & 0 & 4 & 0 & 4 & 4 & 0 & 4 & 0 & 4 & 0 \\
11 & 0 & 0 & 4 & 4 & 0 & 0 & 4 & 0 & 4 & 0 & 0 & 0 & 4 & 4 & 0 & 0 & 0 & 4 & 0 & 4 & 4 & 0 \\
12 & 0 & 4 & 4 & 0 & 0 & 0 & 4 & 0 & 4 & 4 & 0 & 0 & 0 & 0 & 4 & 4 & 0 & 0 & 4 & 0 & 4 & 0 \\
13 & 0 & 4 & 0 & 4 & 0 & 4 & 4 & 0 & 4 & 0 & 4 & 0 & 0 & 0 & 0 & 0 & 4 & 0 & 0 & 0 & 4 & 4 \\
14 & 4 & 4 & 4 & 4 & 0 & 0 & 0 & 4 & 0 & 4 & 4 & 0 & 0 & 0 & 4 & 0 & 0 & 0 & 0 & 0 & 0 & 4 \\
15 & 4 & 4 & 0 & 0 & 0 & 4 & 0 & 0 & 0 & 0 & 0 & 4 & 0 & 4 & 0 & 0 & 4 & 4 & 0 & 4 & 4 & 0 \\
16 & 0 & 0 & 0 & 4 & 4 & 4 & 0 & 0 & 0 & 4 & 0 & 4 & 0 & 0 & 0 & 0 & 4 & 4 & 0 & 4 & 4 & 0 \\
17 & 0 & 4 & 0 & 0 & 0 & 0 & 4 & 0 & 4 & 4 & 0 & 0 & 4 & 0 & 4 & 4 & 0 & 4 & 0 & 0 & 0 & 4 \\
18 & 4 & 4 & 4 & 0 & 0 & 0 & 0 & 0 & 0 & 0 & 4 & 0 & 0 & 0 & 4 & 4 & 4 & 0 & 0 & 4 & 0 & 4 \\
19 & 4 & 0 & 4 & 0 & 0 & 4 & 4 & 0 & 4 & 4 & 0 & 4 & 0 & 0 & 0 & 0 & 0 & 0 & 0 & 4 & 0 & 4 \\
20 & 4 & 0 & 0 & 0 & 4 & 0 & 0 & 4 & 4 & 0 & 4 & 0 & 0 & 0 & 4 & 4 & 0 & 4 & 4 & 0 & 0 & 0 \\
21 & 0 & 0 & 4 & 0 & 4 & 4 & 0 & 0 & 0 & 4 & 4 & 4 & 4 & 0 & 4 & 4 & 0 & 0 & 0 & 0 & 0 & 0 \\
22 & 0 & 0 & 4 & 0 & 4 & 4 & 0 & 4 & 0 & 0 & 0 & 0 & 4 & 4 & 0 & 0 & 4 & 4 & 4 & 0 & 0 & 0
\end{tabular}

The matrix $3 U$ from which $p$ rows and columns are chosen.

placement is less than $1 / 10,000$, even for $k=22$, which tends to decrease as $k$ increases. For larger $k$, it would be too small to apply probabilistic algorithms for finding with a high degree of certainty an optimal selection for each $p$.

The minimum eigenvalue of $W=U / 12$ is -0.4717 , which is a double root of the characteristic equation with the associated eigenvector

$$
\begin{aligned}
& \boldsymbol{x}=\cos (\theta)\left(\begin{array}{llllll}
0.0234 & 0.2942 & 0.2832 & -0.1072 & -0.1651 & 0.0622
\end{array}\right. \\
& \begin{array}{llllll}
0.2295 & -0.2637 & 0.2118 & 0.2732 & -0.0025 & -0.3009
\end{array} \\
& \begin{array}{llllll}
-0.1428 & -0.2263 & 0.1609 & 0.1822 & -0.2981 & -0.2425
\end{array} \\
& \left.\begin{array}{llll}
-0.2753 & 0.0873 & -0.0826 & 0.2988
\end{array}\right)^{\top} \\
& +\sin (\theta)\left(\begin{array}{llllll}
-0.3006 & 0.0660 & -0.1035 & 0.2818 & -0.2523 & 0.2950
\end{array}\right. \\
& 0.1956-0.1462 \quad 0.2146-0.1275-0.30150 .0196 \\
& \begin{array}{llllll}
-0.2656 & 0.1993 & -0.2550 & -0.2402 & 0.0453 & 0.1791
\end{array} \\
& \left.\begin{array}{llll}
-0.1230 & 0.2886 & 0.2900 & 0.0405
\end{array}\right)^{\top} \text {. }
\end{aligned}
$$

Here, the arbitrary constant $\theta$ is chosen to be $3 \pi / 8$, since it maximizes the minimum interval between two adjacent elements in $\boldsymbol{x}$ among several values of $\theta$, and provides 


\begin{tabular}{ccrrl}
$p$ & $\min \chi^{2}$ & $\# \mathcal{K}^{*}(p) \# \mathcal{K}(p)$ & \multicolumn{1}{c}{$S^{*}$} \\
\hline 22 & 0.5714 & 1 & 1 & $\{1, \cdots, 22\}$ \\
21 & 0.5714 & 22 & 22 & $\{1, \cdots, 21\}$ \\
20 & 0.5684 & 132 & 231 & $\{1, \cdots, 20\}$ \\
19 & 0.5614 & 264 & 1,540 & $\{1, \cdots, 19\}$ \\
18 & 0.5490 & 165 & 7,315 & $\{1, \cdots, 13,15,16,17,20,22\}$ \\
17 & 0.5294 & 22 & 26,334 & $\{1, \cdots, 7,9,10,11,12,14,17,18,20,21,22\}$ \\
16 & 0.5111 & 11 & 74,613 & $\{1, \cdots, 7,9,10,12,14,17,18,20,21,22\}$ \\
15 & 0.4952 & 22 & 170,544 & $\{1, \cdots, 5,8,10,11,12,13,15,16,17,19,22\}$ \\
14 & 0.4689 & 11 & 319,770 & $\{1,2,3,4,6,7,9,10,11,15,16,20,21,22\}$ \\
13 & 0.4444 & 44 & 497,420 & $\{1,2,3,4,6,7,9,10,11,15,16,20,22\}$ \\
12 & 0.4040 & 22 & 646,646 & $\{1,2,3,5,6,7,9,10,11,15,16,22\}$ \\
11 & 0.3636 & 22 & 705,432 & $\{1,2,3,5,7,9,10,11,15,16,22\}$ \\
10 & 0.3259 & 22 & 646,646 & $\{1,2,3,5,9,10,11,15,16,22\}$ \\
9 & 0.2963 & 44 & 497,420 & $\{1,2,3,5,10,11,13,15,16\}$ \\
8 & 0.2381 & 11 & 319,770 & $\{1,2,3,10,11,15,16,22\}$ \\
7 & 0.1905 & 22 & 170,544 & $\{1,2,3,10,11,16,22\}$ \\
6 & 0.0889 & 11 & 74,613 & $\{1,2,10,11,16,22\}$ \\
5 & 0 & 22 & 26,334 & $\{1,2,10,11,22\}$ \\
4 & 0 & 165 & 7,315 & $\{1,2,3,10\}$ \\
3 & 0 & 264 & 1,540 & $\{1,2,3\}$ \\
2 & 0 & 132 & 231 & $\{1,2\}$ \\
\hline & & & &
\end{tabular}

Table 3

Summary of the results by using the combinatoric method.

$$
\begin{aligned}
& \boldsymbol{x}=\left(\begin{array}{llllll}
-0.2687 & 0.1735 & 0.0127 & 0.2193 & -0.2963 & 0.2964
\end{array}\right. \\
& \begin{array}{lllllll}
0.2685 & -0.2360 & 0.2793 & -0.0132 & -0.2795 & -0.0971
\end{array} \\
& \begin{array}{llllll}
-0.3000 & 0.0975 & -0.1740 & -0.1522 & -0.0722 & 0.0727
\end{array} \\
& \left.\begin{array}{llll}
-0.2190 & 0.3000 & 0.2363 & 0.1517
\end{array}\right)^{\top}
\end{aligned}
$$

and

$$
Q=\left(\begin{array}{llllllllllllll}
13 & 5 & 11 & 1 & 8 & 19 & 15 & 16 & 12 & 17 & 10 & 3 & \\
& & & 18 & 14 & 22 & 2 & 4 & 21 & 7 & 9 & 6 & 20
\end{array}\right) .
$$

Refinement by using Algorithm 2 with $\alpha=12$ provides

$$
Q^{*}=\left(\begin{array}{llllllllllll}
13 & 8 & 19 & 15 & 16 & 11 & 5 & 1 & 12 & 17 & 3 & 10
\end{array}\right.
$$




\begin{tabular}{|c|c|c|c|c|c|c|c|c|}
\hline$p$ & & & $\begin{array}{l}\mathrm{po} \\
\text { its } \mathrm{r}\end{array}$ & $\begin{array}{l}\text { le vi } \\
\text { p of }\end{array}$ & of $\lambda$ & & & \\
\hline 22 & $\begin{array}{c}0.5714 \\
100 \%\end{array}$ & & & & & & & \\
\hline 21 & $\begin{array}{c}0.5714 \\
100 \%\end{array}$ & & & & & & & \\
\hline 20 & $\begin{array}{c}0.5684 \\
57 \%\end{array}$ & $\begin{array}{c}0.5754 \\
43 \%\end{array}$ & & & & & & \\
\hline 19 & $\begin{array}{c}0.5614 \\
17 \%\end{array}$ & $\begin{array}{c}0.5692 \\
43 \%\end{array}$ & $\begin{array}{c}0.577 \\
34 \%\end{array}$ & $\begin{array}{c}0.5848 \\
6 \%\end{array}$ & & & & \\
\hline 18 & $\begin{array}{c}0.549 \\
2 \%\end{array}$ & $\begin{array}{c}0.5577 \\
14 \%\end{array}$ & $\begin{array}{c}0.5664 \\
31 \%\end{array}$ & $\begin{array}{c}0.5752 \\
33 \%\end{array}$ & $\begin{array}{c}0.5839 \\
17 \%\end{array}$ & $\begin{array}{c}0.5926 \\
3 \%\end{array}$ & $\begin{array}{c}0.6013 \\
0.2 \%\end{array}$ & \\
\hline 17 & $\begin{array}{l}0.5294 \\
0.08 \%\end{array}$ & $\begin{array}{c}0.5392 \\
1 \%\end{array}$ & $\begin{array}{c}0.549 \\
8 \%\end{array}$ & $\begin{array}{c}0.5588 \\
18 \%\end{array}$ & $\begin{array}{c}0.5686 \\
29 \%\end{array}$ & $\begin{array}{c}0.5784 \\
25 \%\end{array}$ & $\begin{array}{c}0.5882 \\
15 \%\end{array}$ & $\begin{array}{l}\geq 0.598 \\
4 \%\end{array}$ \\
\hline 16 & $\begin{array}{l}0.5111 \\
0.01 \%\end{array}$ & $\begin{array}{c}0.5222 \\
0.4 \%\end{array}$ & $\begin{array}{c}0.5333 \\
2 \%\end{array}$ & $\begin{array}{c}0.5444 \\
8 \%\end{array}$ & $\begin{array}{c}0.5556 \\
16 \%\end{array}$ & $\begin{array}{c}0.5667 \\
26 \%\end{array}$ & $\begin{array}{c}0.5778 \\
23 \%\end{array}$ & $\begin{array}{l}\geq 0.5889 \\
25 \%\end{array}$ \\
\hline 15 & $\begin{array}{l}0.4952 \\
0.01 \%\end{array}$ & $\begin{array}{c}0.5079 \\
0.4 \%\end{array}$ & $\begin{array}{c}0.5206 \\
2 \%\end{array}$ & $\begin{array}{c}0.5333 \\
5 \%\end{array}$ & $\begin{array}{l}0.546 \\
12 \%\end{array}$ & $\begin{array}{c}0.5587 \\
19 \%\end{array}$ & $\begin{array}{c}0.5714 \\
23 \%\end{array}$ & $\begin{aligned} & \geq 0.5841 \\
& 39 \%\end{aligned}$ \\
\hline 14 & $\begin{array}{l}0.4689 \\
0.003 \%\end{array}$ & $\begin{array}{l}0.4835 \\
0.09 \%\end{array}$ & $\begin{array}{c}0.4982 \\
0.7 \%\end{array}$ & $\begin{array}{c}0.5128 \\
2 \%\end{array}$ & $\begin{array}{c}0.5275 \\
6 \%\end{array}$ & $\begin{array}{c}0.5421 \\
12 \%\end{array}$ & $\begin{array}{c}0.5568 \\
19 \%\end{array}$ & $\begin{array}{l}\geq 0.5714 \\
61 \%\end{array}$ \\
\hline 13 & $\begin{array}{c}0.4444 \\
0.009 \%\end{array}$ & $\begin{array}{l}0.4615 \\
0.09 \%\end{array}$ & $\begin{array}{c}0.4786 \\
0.4 \%\end{array}$ & $\begin{array}{c}0.4957 \\
1 \%\end{array}$ & $\begin{array}{c}0.5128 \\
5 \%\end{array}$ & $\begin{array}{c}0.5299 \\
9 \%\end{array}$ & $\begin{array}{l}0.547 \\
16 \%\end{array}$ & $\begin{array}{l}\geq 0.5641 \\
68 \%\end{array}$ \\
\hline 12 & $\begin{array}{c}0.404 \\
0.003 \%\end{array}$ & $\begin{array}{l}0.4242 \\
0.03 \%\end{array}$ & $\begin{array}{c}0.4444 \\
0.1 \%\end{array}$ & $\begin{array}{c}0.4646 \\
0.6 \%\end{array}$ & $\begin{array}{c}0.4848 \\
2 \%\end{array}$ & $\begin{array}{c}0.5051 \\
5 \%\end{array}$ & $\begin{array}{c}0.5253 \\
10 \%\end{array}$ & $\begin{aligned} \geq & 0.5455 \\
& 82 \%\end{aligned}$ \\
\hline 11 & $\begin{array}{c}0.3636 \\
0.003 \%\end{array}$ & $\begin{array}{l}0.3879 \\
0.02 \%\end{array}$ & $\begin{array}{l}0.4121 \\
0.09 \%\end{array}$ & $\begin{array}{c}0.4364 \\
0.4 \%\end{array}$ & $\begin{array}{c}0.4606 \\
2 \%\end{array}$ & $\begin{array}{c}0.4848 \\
4 \%\end{array}$ & $\begin{array}{c}0.5091 \\
9 \%\end{array}$ & $\begin{aligned} & \geq 0.5333 \\
& 85 \%\end{aligned}$ \\
\hline 10 & $\begin{array}{c}0.3259 \\
0.003 \%\end{array}$ & $\begin{array}{l}0.3556 \\
0.03 \%\end{array}$ & $\begin{array}{c}0.3852 \\
0.1 \%\end{array}$ & $\begin{array}{c}0.4148 \\
0.6 \%\end{array}$ & $\begin{array}{c}0.4444 \\
2 \%\end{array}$ & $\begin{array}{c}0.4741 \\
5 \%\end{array}$ & $\begin{array}{c}0.5037 \\
10 \%\end{array}$ & $\begin{aligned} \geq & 0.5333 \\
& 82 \%\end{aligned}$ \\
\hline 9 & $\begin{array}{l}0.2963 \\
0.009 \%\end{array}$ & $\begin{array}{l}0.3333 \\
0.09 \%\end{array}$ & $\begin{array}{c}0.3704 \\
0.4 \%\end{array}$ & $\begin{array}{c}0.4074 \\
1 \%\end{array}$ & $\begin{array}{c}0.4444 \\
5 \%\end{array}$ & $\begin{array}{c}0.4815 \\
9 \%\end{array}$ & $\begin{array}{c}0.5185 \\
16 \%\end{array}$ & $\begin{array}{l}\geq 0.5556 \\
68 \%\end{array}$ \\
\hline 8 & $\begin{array}{l}0.2381 \\
0.003 \%\end{array}$ & $\begin{array}{l}0.2857 \\
0.09 \%\end{array}$ & $\begin{array}{c}0.3333 \\
0.7 \%\end{array}$ & $\begin{array}{c}0.381 \\
2 \%\end{array}$ & $\begin{array}{c}0.4286 \\
6 \%\end{array}$ & $\begin{array}{c}0.4762 \\
12 \%\end{array}$ & $\begin{array}{c}0.5238 \\
19 \%\end{array}$ & $\begin{array}{c}\geq 0.5714 \\
61 \%\end{array}$ \\
\hline 7 & $\begin{array}{l}0.1905 \\
0.01 \%\end{array}$ & $\begin{array}{l}0.254 \\
0.4 \%\end{array}$ & $\begin{array}{c}0.3175 \\
2 \%\end{array}$ & $\begin{array}{c}0.381 \\
5 \%\end{array}$ & $\begin{array}{c}0.4444 \\
12 \%\end{array}$ & $\begin{array}{c}0.5079 \\
19 \%\end{array}$ & $\begin{array}{c}0.5714 \\
23 \%\end{array}$ & $\begin{aligned} \geq & 0.6349 \\
& 39 \%\end{aligned}$ \\
\hline 6 & $\begin{array}{l}0.0889 \\
0.01 \%\end{array}$ & $\begin{array}{c}0.1778 \\
0.4 \%\end{array}$ & $\begin{array}{c}0.2667 \\
2 \%\end{array}$ & $\begin{array}{c}0.3556 \\
8 \%\end{array}$ & $\begin{array}{c}0.4444 \\
16 \%\end{array}$ & $\begin{array}{c}0.5333 \\
26 \%\end{array}$ & $\begin{array}{c}0.6222 \\
23 \%\end{array}$ & $\begin{array}{l}\geq 0.7111 \\
25 \%\end{array}$ \\
\hline 5 & $\begin{array}{c}0 \\
0.08 \%\end{array}$ & $\begin{array}{c}0.1333 \\
1 \%\end{array}$ & $\begin{array}{c}0.2667 \\
8 \%\end{array}$ & $\begin{array}{c}0.4 \\
18 \%\end{array}$ & $\begin{array}{c}0.5333 \\
29 \%\end{array}$ & $\begin{array}{c}0.6667 \\
25 \%\end{array}$ & $\begin{array}{c}0.8 \\
15 \%\end{array}$ & $\begin{array}{c}\geq 0.9333 \\
4 \%\end{array}$ \\
\hline 4 & $\begin{array}{c}0 \\
2 \%\end{array}$ & $\begin{array}{c}0.2222 \\
14 \%\end{array}$ & $\begin{array}{c}0.4444 \\
31 \%\end{array}$ & $\begin{array}{c}0.6667 \\
33 \%\end{array}$ & $\begin{array}{c}0.8889 \\
17 \%\end{array}$ & $\begin{array}{c}1.1111 \\
3 \%\end{array}$ & $\begin{array}{c}1.3333 \\
0.2 \%\end{array}$ & \\
\hline 3 & $\begin{array}{c}0 \\
17 \%\end{array}$ & $\begin{array}{c}0.4444 \\
43 \%\end{array}$ & $\begin{array}{c}0.8889 \\
34 \%\end{array}$ & $\begin{array}{c}1.3333 \\
6 \%\end{array}$ & & & & \\
\hline 2 & $\begin{array}{c}0 \\
57 \%\end{array}$ & $\begin{array}{c}1.3333 \\
43 \%\end{array}$ & & & & & & \\
\hline
\end{tabular}

Table 4

All the possible values of $\chi^{2}$ and their ratios of appearance.

$$
\left.\begin{array}{llllllllll}
18 & 14 & 7 & 6 & 9 & 22 & 2 & 4 & 21 & 20
\end{array}\right),
$$

and furnishes the optimal mean $\chi^{2}$ 's for every $p \in[2,22]$. More precisely, the set composed of the first $p$ elements of $Q^{*}$ provides the optimal selection of columns from $D$, i.e.,

$$
\{13,8\} \in \mathcal{K}^{*}(2),\{13,8,19\} \in \mathcal{K}^{*}(3),\{13,8,19,15\} \in \mathcal{K}^{*}(4),
$$


Table 5

\begin{tabular}{r|lllllllllllllllllllllll} 
& 13 & 5 & 11 & 1 & 8 & 191516121710 & 18 & 161422 & 2 & 4 & 1 & 7 & 9 & 6 & 20 \\
\hline 13 & 0 & 0 & 4 & 0 & 0 & 0 & 0 & 0 & 0 & 4 & 0 & 0 & 0 & 0 & 4 & 4 & 4 & 4 & 4 & 4 & 4 & 0 \\
5 & 0 & 0 & 0 & 0 & 4 & 0 & 0 & 4 & 0 & 0 & 0 & 0 & 0 & 0 & 4 & 4 & 4 & 4 & 4 & 0 & 4 & 4 \\
11 & 4 & 0 & 0 & 0 & 0 & 0 & 0 & 0 & 0 & 0 & 0 & 4 & 4 & 4 & 0 & 0 & 4 & 4 & 4 & 4 & 0 & 4 \\
1 & 0 & 0 & 0 & 0 & 0 & 4 & 4 & 0 & 0 & 0 & 0 & 0 & 4 & 4 & 0 & 0 & 4 & 0 & 4 & 4 & 4 & 4 \\
8 & 0 & 4 & 0 & 0 & 0 & 0 & 0 & 0 & 0 & 0 & 4 & 4 & 0 & 4 & 4 & 4 & 0 & 0 & 0 & 4 & 4 & 4 \\
19 & 0 & 0 & 0 & 4 & 0 & 0 & 0 & 0 & 4 & 0 & 4 & 4 & 0 & 0 & 4 & 0 & 0 & 0 & 4 & 4 & 4 & 4 \\
15 & 0 & 0 & 0 & 4 & 0 & 0 & 0 & 0 & 4 & 4 & 0 & 0 & 4 & 4 & 0 & 4 & 0 & 4 & 0 & 0 & 4 & 4 \\
16 & 0 & 4 & 0 & 0 & 0 & 0 & 0 & 0 & 4 & 4 & 4 & 0 & 4 & 0 & 0 & 0 & 4 & 4 & 0 & 0 & 4 & 4 \\
12 & 0 & 0 & 0 & 0 & 0 & 4 & 4 & 4 & 0 & 0 & 4 & 4 & 0 & 0 & 0 & 4 & 0 & 4 & 4 & 4 & 0 & 0 \\
17 & 4 & 0 & 0 & 0 & 0 & 0 & 4 & 4 & 0 & 0 & 4 & 0 & 4 & 0 & 4 & 4 & 0 & 0 & 4 & 4 & 0 & 0 \\
10 & 0 & 0 & 0 & 0 & 4 & 4 & 0 & 4 & 4 & 4 & 0 & 0 & 0 & 4 & 0 & 0 & 4 & 4 & 0 & 4 & 0 & 0 \\
3 & 0 & 0 & 4 & 0 & 4 & 4 & 0 & 0 & 4 & 0 & 0 & 0 & 4 & 4 & 4 & 0 & 4 & 4 & 0 & 0 & 0 & 0 \\
18 & 0 & 0 & 4 & 4 & 0 & 0 & 4 & 4 & 0 & 4 & 0 & 4 & 0 & 0 & 4 & 4 & 0 & 0 & 0 & 0 & 0 & 4 \\
14 & 0 & 0 & 4 & 4 & 4 & 0 & 4 & 0 & 0 & 0 & 4 & 4 & 0 & 0 & 4 & 4 & 4 & 0 & 0 & 0 & 0 & 0 \\
22 & 4 & 4 & 0 & 0 & 4 & 4 & 0 & 0 & 0 & 4 & 0 & 4 & 4 & 4 & 0 & 0 & 0 & 0 & 0 & 0 & 4 & 0 \\
2 & 4 & 4 & 0 & 0 & 4 & 0 & 4 & 0 & 4 & 4 & 0 & 0 & 4 & 4 & 0 & 0 & 0 & 0 & 4 & 0 & 0 & 0 \\
4 & 4 & 4 & 4 & 4 & 0 & 0 & 0 & 4 & 0 & 0 & 4 & 4 & 0 & 4 & 0 & 0 & 0 & 0 & 4 & 0 & 0 & 0 \\
21 & 4 & 4 & 4 & 0 & 0 & 0 & 4 & 4 & 4 & 0 & 4 & 4 & 0 & 0 & 0 & 0 & 0 & 0 & 0 & 0 & 4 & 0 \\
7 & 4 & 4 & 4 & 4 & 0 & 4 & 0 & 0 & 4 & 4 & 0 & 0 & 0 & 0 & 0 & 4 & 4 & 0 & 0 & 0 & 0 & 0 \\
9 & 4 & 0 & 4 & 4 & 4 & 4 & 0 & 0 & 4 & 4 & 4 & 0 & 0 & 0 & 0 & 0 & 0 & 0 & 0 & 0 & 0 & 4 \\
6 & 4 & 4 & 0 & 4 & 4 & 4 & 4 & 4 & 0 & 0 & 0 & 0 & 0 & 0 & 4 & 0 & 0 & 4 & 0 & 0 & 0 & 0 \\
20 & 0 & 4 & 4 & 4 & 4 & 4 & 4 & 4 & 0 & 0 & 0 & 0 & 4 & 0 & 0 & 0 & 0 & 0 & 0 & 4 & 0 & 0
\end{tabular}

Diagonalized matrix $3 U^{Q}$ by statistical ordering.

Table 6

\begin{tabular}{r|llllllllllllllllllllllll} 
& 13 & 8 & 19151611 & 1 & 1 & 1217 & 3 & 101814 & 7 & 6 & 9 & 22 & 2 & 4 & 2120 \\
\hline 1 & 1 & 1 & 2 & 2 & 1 & 2 & 2 & 1 & 1 & 2 & 1 & 2 & 1 & 2 & 2 & 1 & 1 & 2 & 1 & 1 & 2 & 2 \\
2 & 2 & 1 & 2 & 1 & 2 & 1 & 1 & 1 & 1 & 1 & 1 & 2 & 2 & 2 & 1 & 2 & 2 & 1 & 1 & 2 & 2 & 2 \\
3 & 2 & 2 & 2 & 2 & 1 & 1 & 2 & 1 & 2 & 2 & 2 & 1 & 2 & 1 & 1 & 1 & 2 & 1 & 1 & 1 & 1 & 2 \\
4 & 1 & 2 & 2 & 1 & 2 & 2 & 1 & 1 & 2 & 2 & 1 & 1 & 2 & 2 & 2 & 1 & 1 & 1 & 2 & 2 & 1 & 1 \\
5 & 2 & 1 & 1 & 2 & 2 & 2 & 1 & 2 & 1 & 2 & 2 & 2 & 2 & 1 & 2 & 2 & 1 & 1 & 1 & 1 & 1 & 1 \\
6 & 2 & 2 & 1 & 2 & 2 & 2 & 2 & 2 & 1 & 1 & 1 & 1 & 1 & 2 & 1 & 1 & 2 & 2 & 1 & 2 & 1 & 1 \\
7 & 2 & 1 & 1 & 1 & 1 & 2 & 1 & 1 & 2 & 1 & 2 & 1 & 1 & 2 & 2 & 2 & 2 & 2 & 2 & 1 & 1 & 2 \\
8 & 2 & 2 & 2 & 1 & 1 & 2 & 2 & 2 & 2 & 1 & 1 & 2 & 1 & 1 & 1 & 2 & 1 & 1 & 2 & 1 & 2 & 1 \\
9 & 1 & 2 & 1 & 1 & 2 & 1 & 2 & 1 & 1 & 1 & 2 & 2 & 2 & 1 & 2 & 1 & 2 & 2 & 2 & 1 & 2 & 1 \\
10 & 1 & 2 & 1 & 2 & 1 & 1 & 1 & 2 & 1 & 2 & 1 & 1 & 1 & 1 & 2 & 2 & 2 & 1 & 2 & 2 & 2 & 2 \\
11 & 1 & 1 & 1 & 1 & 1 & 1 & 2 & 2 & 2 & 2 & 2 & 1 & 2 & 2 & 1 & 2 & 1 & 2 & 1 & 2 & 2 & 1 \\
12 & 1 & 1 & 2 & 2 & 2 & 1 & 1 & 2 & 2 & 1 & 2 & 2 & 1 & 1 & 1 & 1 & 1 & 2 & 2 & 2 & 1 & 2
\end{tabular}

The design $D^{Q^{*}}$ with permuted columns.

$$
\cdots \quad\{13,8,19,15,16, \ldots, 20\} \in \mathcal{K}^{*}(22) .
$$

Experimental factors should be assigned to the columns $13,8,19,15, \ldots, 20$ of the original design matrix $D$ in this order, or, simply to the columns of the permuted design matrix $D^{Q^{*}}$ shown in Table 6 from the left. The corresponding strictly optimal $\chi^{2}$ matrix $U^{Q^{*}}$ with permuted rows and columns of $U$ is shown in Table 7. 
Table 7

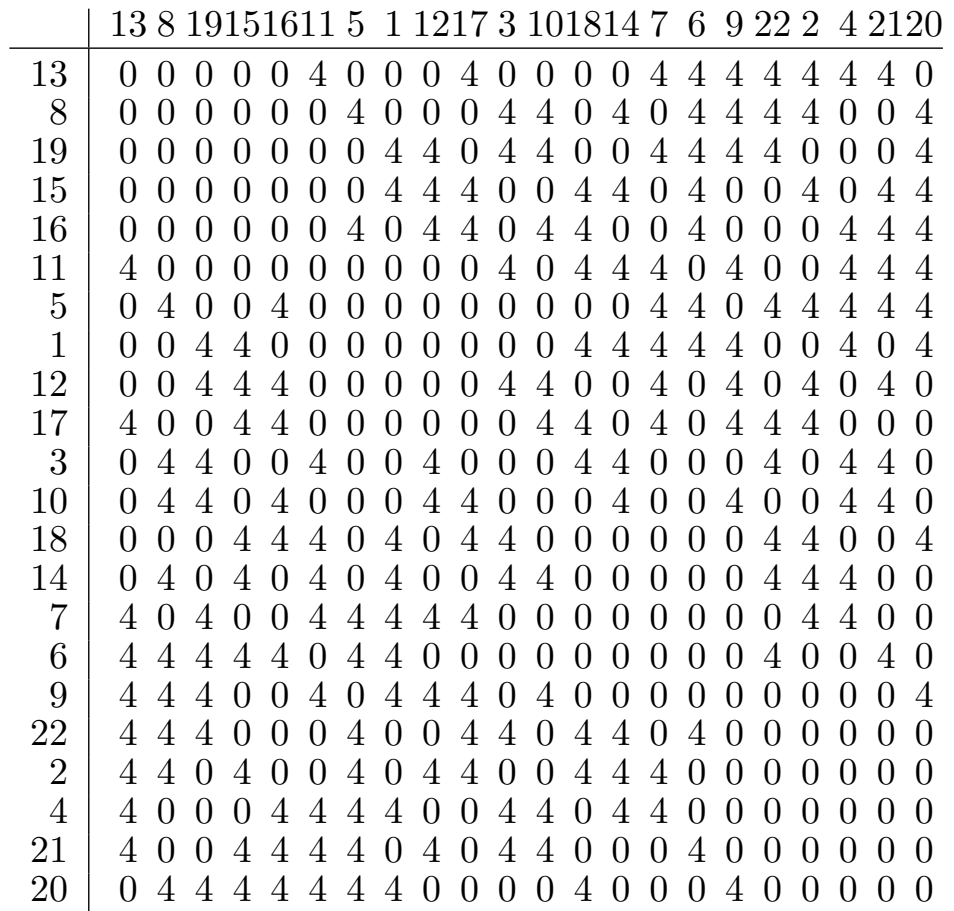

The ordered matrix $3 U^{Q^{*}}$ with strict optimality.

\subsection{Two trivial cases}

Special treatments may sometimes be required in ordering columns of supersaturated designs with simple or decomposable structures.

The design shown in Tables 8 is composed from an Hadamard matrix for $t=2$, $n=10$ and $k=18$ (Lin, 1993) and has a strictly optimal $U^{Q}$ provided in Table 9 after the permutation of columns with

$$
Q=(1,2,3,4,6,7,9,11,14,16,12,15,18,10,13,17,5,8) .
$$

The eigenvalues of the equation (20) are -0.36 (9-tuple root), 0.28 (8-tuple) and 1. The set $\left\{\boldsymbol{e}_{i}-\boldsymbol{e}_{j} \mid(i, j) \in P\right\}$ of vectors are an orthogonal base of eigenspace corresponding to -0.36 , where $\boldsymbol{e}_{i}$ is the unit vector of which the $i$-th element is 1 and

$$
\begin{aligned}
P=\{(1,8),(2,5), & (3,17),(4,13),(6,10), \\
& (7,18),(9,15),(11,12),(14,16)\} .
\end{aligned}
$$

In other words, the optimal ordering in this case simply finds the pairs in $P$ of columns to be placed symmetrically as in (29), which is archived by choosing a vector with distinct elements from the eigenspace, for example, 
Table 8

\begin{tabular}{|c|c|}
\hline & 23345667 \\
\hline 1 & $\begin{array}{lllllllllllllllll}1 & 2 & 2 & 1 & 1 & 1 & 1 & 2 & 1 & 2 & 1 & 2 & 2 & 2 & 2 & 1 & 1\end{array}$ \\
\hline 2 & $\begin{array}{lllllllllllllllll}2 & 2 & 1 & 1 & 1 & 1 & 2 & 1 & 2 & 1 & 2 & 2 & 2 & 2 & 1 & 1 & 2\end{array}$ \\
\hline 3 & $\begin{array}{lllllllllllll} & 1 & 2 & 1 & 2 & 2 & 2 & 2 & 1 & 1 & 2 & 1 & 1\end{array}$ \\
\hline 4 & $\begin{array}{lllllllll}2 & 2 & 2 & 1 & 1 & 2 & 1 & 1 & 2\end{array}$ \\
\hline 5 & $\begin{array}{llllllllll}2 & 2 & 1 & 1 & 2 & 1 & 1 & 2 & 2 & 1\end{array}$ \\
\hline 6 & $\begin{array}{lllllllllll}2 & 2 & 1 & 1 & 2 & 1 & 1 & 2 & 2 & 1\end{array}$ \\
\hline 7 & $\begin{array}{llllll}2 & 1 & 1 & 2 & 2 & 1\end{array}$ \\
\hline 8 & 11112 \\
\hline 9 & $\begin{array}{lllllll}1 & 1 & 2 & 2 & 1\end{array}$ \\
\hline 10 & \\
\hline
\end{tabular}

A supersaturated design $(t=2, n=10, k=18)$, Lin (1993).

Table 9

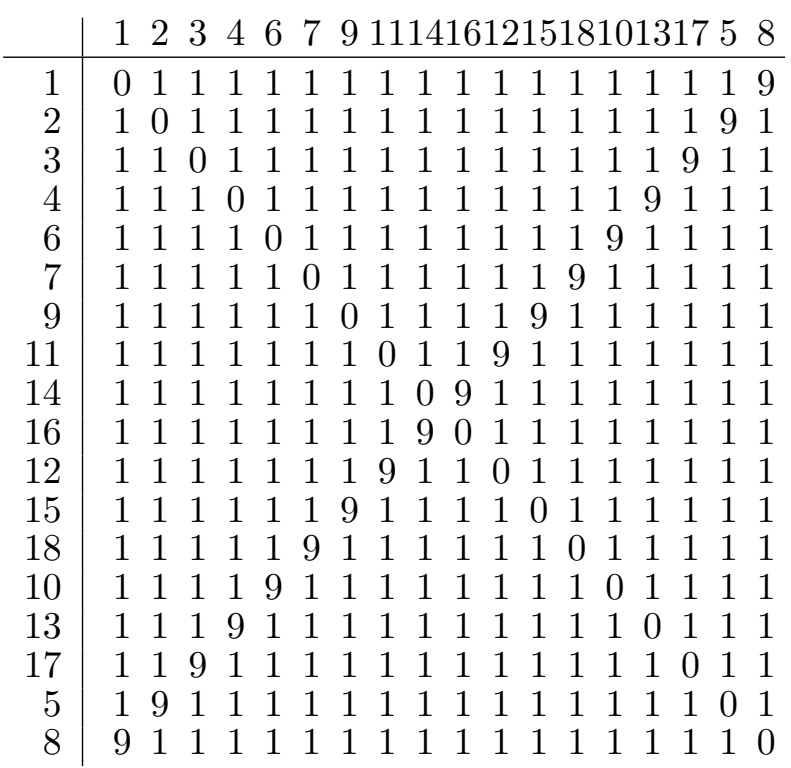

An optimally permuted matrix $(5 / 2) U^{Q}$.

$\sum_{(i, j) \in P}(i-15)\left(\boldsymbol{e}_{i}-\boldsymbol{e}_{j}\right)$

Table 10 shows another supersaturated design by Lin (1993) with $t=2, n=14$ and $k=26$.

In this case, the eigenvalues of the equation (20) are $-0.51,-0.36$ (6-tuple), -0.16 (6-tuple), 0.12 (6-tuple), 0.32 (6-tuple) and 1. The corresponding eigenvector to -0.51 , the simple smallest root, is $\sum_{i \in G_{1}} \boldsymbol{e}_{i}-\sum_{i \in G_{2}} \boldsymbol{e}_{i}$, where

$$
\begin{aligned}
& G_{1}=\{1,3,4,6,8,9,10,13,17,22,23,24,25\}, \\
& G_{2}=\{2,5,7,11,12,14,15,16,18,19,20,21,26\} .
\end{aligned}
$$

The above results imply that grouping the columns into $G_{1}$ or $G_{2}$ is essential. In fact, Table 11 demonstrates that permutation of columns within a group does not affect any $\chi^{2}$ value, where the ordinates provided by 
Table 10

\begin{tabular}{r|llllllllllllllllllllllllllllll} 
& 1 & 2 & 3 & 4 & 5 & 6 & 7 & 8 & 9 & 10111 & 1314 & 151617181920 & 212223242 & 526 \\
\hline 1 & 1 & 2 & 1 & 1 & 1 & 1 & 2 & 2 & 2 & 2 & 1 & 2 & 2 & 2 & 1 & 2 & 2 & 1 & 1 & 1 & 2 & 1 & 2 & 1 & 1 & 2 \\
2 & 2 & 1 & 1 & 1 & 1 & 1 & 2 & 2 & 2 & 1 & 2 & 2 & 2 & 1 & 2 & 2 & 1 & 2 & 1 & 2 & 1 & 2 & 1 & 1 & 2 & 1 \\
3 & 2 & 2 & 2 & 1 & 2 & 1 & 1 & 1 & 1 & 2 & 2 & 1 & 2 & 1 & 2 & 2 & 2 & 1 & 1 & 2 & 1 & 1 & 1 & 2 & 1 & 2 \\
4 & 2 & 2 & 2 & 2 & 1 & 1 & 1 & 1 & 1 & 2 & 1 & 2 & 1 & 2 & 2 & 2 & 1 & 2 & 2 & 1 & 1 & 1 & 2 & 1 & 2 & 1 \\
5 & 1 & 1 & 1 & 2 & 2 & 2 & 1 & 1 & 2 & 1 & 2 & 2 & 1 & 2 & 2 & 2 & 2 & 1 & 1 & 1 & 2 & 1 & 1 & 2 & 2 & 1 \\
6 & 1 & 1 & 1 & 2 & 2 & 2 & 2 & 1 & 1 & 2 & 1 & 2 & 2 & 1 & 2 & 1 & 2 & 2 & 2 & 1 & 1 & 2 & 1 & 1 & 1 & 2 \\
7 & 1 & 1 & 2 & 1 & 2 & 1 & 1 & 2 & 1 & 1 & 2 & 1 & 1 & 1 & 1 & 2 & 2 & 2 & 2 & 1 & 2 & 2 & 2 & 1 & 2 & 2 \\
8 & 1 & 2 & 1 & 1 & 1 & 2 & 1 & 2 & 1 & 2 & 2 & 2 & 1 & 2 & 1 & 1 & 1 & 1 & 2 & 2 & 1 & 2 & 1 & 2 & 2 & 2 \\
9 & 1 & 1 & 2 & 1 & 1 & 2 & 2 & 1 & 1 & 1 & 1 & 1 & 2 & 2 & 2 & 1 & 1 & 2 & 1 & 2 & 2 & 1 & 2 & 2 & 2 & 2 \\
10 & 2 & 2 & 1 & 2 & 1 & 2 & 2 & 2 & 1 & 1 & 2 & 1 & 1 & 1 & 2 & 1 & 2 & 1 & 2 & 2 & 2 & 1 & 2 & 1 & 1 & 1 \\
11 & 1 & 2 & 2 & 2 & 2 & 1 & 1 & 2 & 2 & 1 & 1 & 2 & 2 & 1 & 1 & 1 & 1 & 2 & 2 & 2 & 2 & 1 & 1 & 2 & 1 & 1 \\
12 & 2 & 1 & 2 & 1 & 2 & 2 & 2 & 1 & 2 & 2 & 1 & 1 & 1 & 2 & 1 & 2 & 1 & 1 & 2 & 2 & 2 & 2 & 1 & 1 & 1 & 1 \\
13 & 2 & 2 & 1 & 2 & 2 & 1 & 2 & 1 & 2 & 1 & 2 & 1 & 1 & 2 & 1 & 1 & 1 & 2 & 1 & 1 & 1 & 2 & 2 & 2 & 1 & 2 \\
14 & 2 & 1 & 2 & 2 & 1 & 2 & 1 & 2 & 2 & 2 & 1 & 1 & 2 & 1 & 1 & 1 & 2 & 1 & 1 & 1 & 1 & 2 & 2 & 2 & 2 & 1
\end{tabular}

A supersaturated design $(t=2, n=14, k=26)$, Lin (1993).

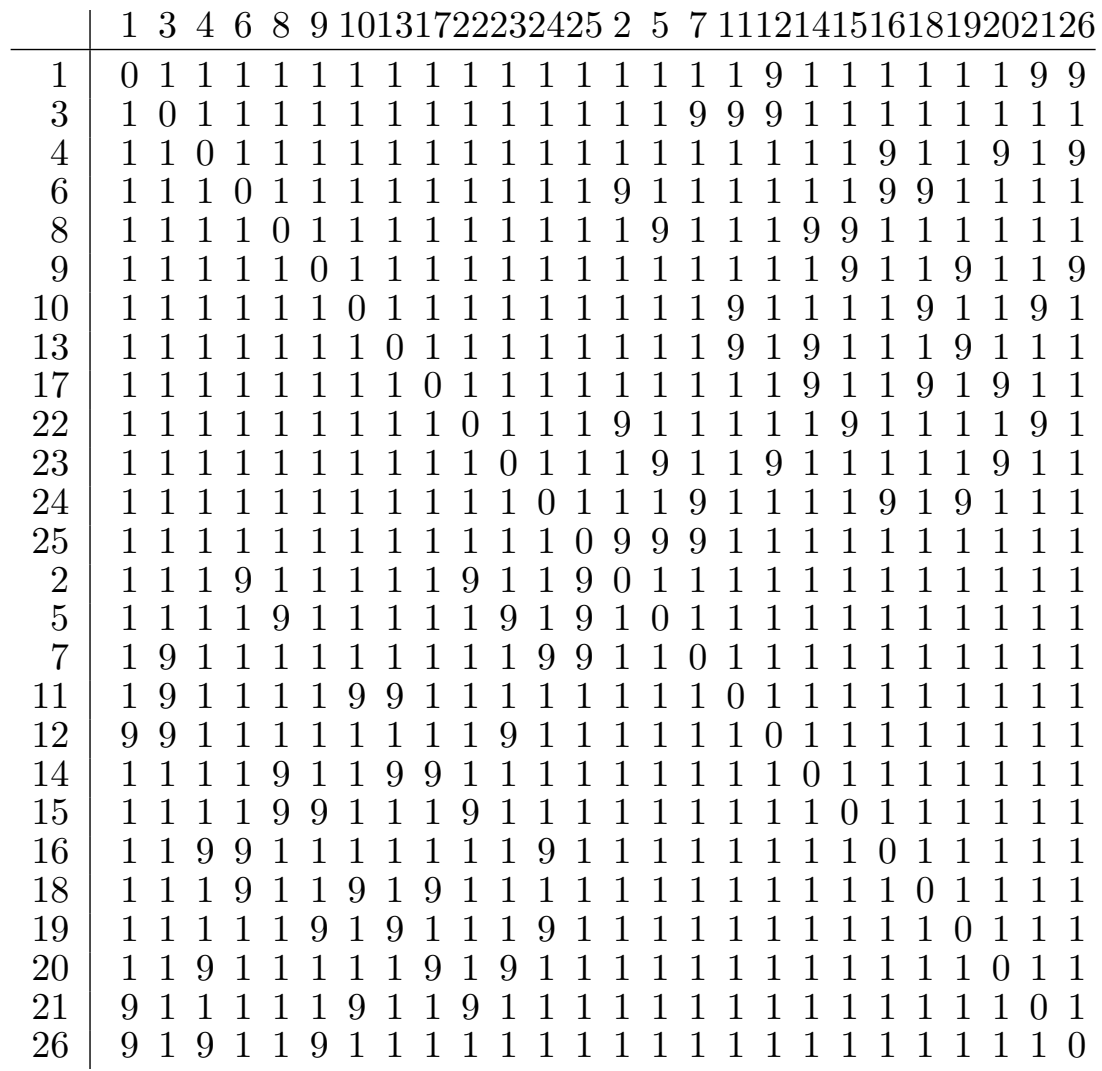

Table 11

An optimally permuted matrix $(7 / 2) U^{Q}$.

$$
-100\left(\sum_{i \in G_{1}} \boldsymbol{e}_{i}-\sum_{i \in G_{2}} \boldsymbol{e}_{i}\right)+j \boldsymbol{e}_{j} \quad(j=1, \ldots, 26)
$$

yield a representative element of the strictly optimal permutations. 


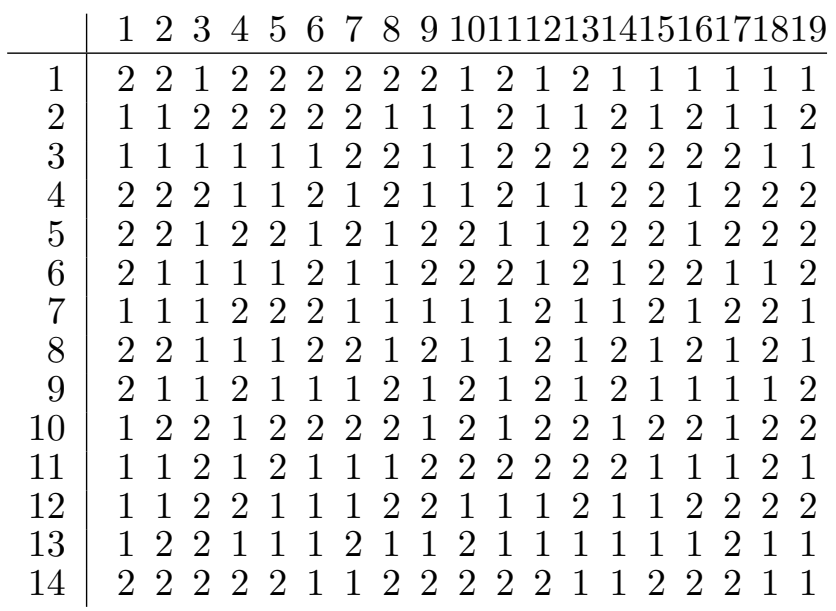

Table 12

An $\mathrm{E}\left(s^{2}\right)$-optimal design for $n=14$ and $k=19$, Bulutoglu and Cheng (2004).

\subsection{Ordination of $\mathrm{E}\left(s^{2}\right)$-optimal designs}

For E $\left(s^{2}\right)$ criterion, Bulutoglu and Cheng (2004) have provided a precise lower bound $\mathrm{E}(n, k)$ to the $\mathrm{E}\left(s^{2}\right)$ 's for $n \times k$ designs. A design that satisfies $\mathrm{E}\left(s^{2}\right)=$ $\mathrm{E}(n, k)$ is referred to as being $\mathrm{E}\left(s^{2}\right)$-optimal.

Table 12 shows an E( $\left.s^{2}\right)$-optimal design by Bulutoglu and Cheng (2004) for $n=14$ and $k=19$ with $\mathrm{E}\left(s^{2}\right)=14 \chi^{2}(K)=6.0585$.

The statistical ordering provides $U^{Q}$ as shown in Table 13 which reveals that columns 8, 14 and 16 are superior and the first column is inferior in mutual orthogonality. Re-ordering of the other columns and some heuristic optimization yield an optimal permutation $Q^{*}$ of columns shown in Table 14, which may be useful in the ordination of $\mathrm{E}\left(s^{2}\right)$-optimal designs as follows:

(1) The structure of a design when considering dependency is visually shown using $U^{Q}$ or $U^{Q^{*}}$. This may help users of the design e.g., in allocating promising factors to the columns with preferable properties.

(2) The first to eighteenth columns of $Q^{*}$ have

$$
\mathrm{E}\left(s^{2}\right)=14 \chi^{2}(K-\{1\})=5.67320=\underline{\mathrm{E}}(14,18),
$$

which means that an optimal design for $k=18$ is implanted in this design. In other words, an $\mathrm{E}\left(s^{2}\right)$-optimal design with ordinated columns may concurrently serve as one or more optimal or suboptimal designs with lesser numbers of columns.

(3) It is worth pointing out that the preceding fact implies the possibility that unknown $\mathrm{E}\left(s^{2}\right)$-optimal designs will be discovered by the ordination of the columns. 
Table 13

\begin{tabular}{r|lllllllllllllllllll} 
& 1 & 7 & 1316 & 8 & 14 & 51018 & 5 & 1219 & 4 & 11 & 6 & 17 & 3 & 9 & 2 \\
\hline 1 & 0 & 1 & 1 & 1 & 1 & 1 & 1 & 1 & 1 & 1 & 1 & 1 & 1 & 1 & 1 & 1 & 9 & 9 & 9 \\
7 & 1 & 0 & 1 & 1 & 1 & 1 & 1 & 1 & 1 & 1 & 1 & 1 & 1 & 1 & 1 & 1 & 1 & 1 & 9 \\
13 & 1 & 1 & 0 & 1 & 1 & 1 & 1 & 1 & 1 & 1 & 1 & 1 & 1 & 1 & 1 & 1 & 1 & 9 & 1 \\
16 & 1 & 1 & 1 & 0 & 1 & 1 & 1 & 1 & 1 & 1 & 1 & 1 & 1 & 1 & 1 & 1 & 1 & 1 & 1 \\
8 & 1 & 1 & 1 & 1 & 0 & 1 & 1 & 1 & 1 & 1 & 1 & 1 & 1 & 1 & 1 & 1 & 1 & 1 & 1 \\
14 & 1 & 1 & 1 & 1 & 1 & 0 & 1 & 1 & 1 & 1 & 1 & 1 & 1 & 1 & 1 & 1 & 1 & 1 & 1 \\
15 & 1 & 1 & 1 & 1 & 1 & 1 & 0 & 1 & 1 & 1 & 1 & 1 & 1 & 1 & 1 & 9 & 1 & 1 & 1 \\
10 & 1 & 1 & 1 & 1 & 1 & 1 & 1 & 0 & 1 & 1 & 1 & 1 & 1 & 1 & 9 & 1 & 1 & 1 & 1 \\
18 & 1 & 1 & 1 & 1 & 1 & 1 & 1 & 1 & 0 & 1 & 1 & 1 & 1 & 9 & 1 & 1 & 1 & 1 & 1 \\
5 & 1 & 1 & 1 & 1 & 1 & 1 & 1 & 1 & 1 & 0 & 1 & 1 & 9 & 1 & 1 & 1 & 1 & 1 & 1 \\
12 & 1 & 1 & 1 & 1 & 1 & 1 & 1 & 1 & 1 & 1 & 0 & 9 & 1 & 1 & 1 & 1 & 1 & 1 & 1 \\
19 & 1 & 1 & 1 & 1 & 1 & 1 & 1 & 1 & 1 & 1 & 9 & 0 & 1 & 1 & 1 & 1 & 1 & 1 & 1 \\
4 & 1 & 1 & 1 & 1 & 1 & 1 & 1 & 1 & 1 & 9 & 1 & 1 & 0 & 1 & 1 & 1 & 1 & 1 & 1 \\
11 & 1 & 1 & 1 & 1 & 1 & 1 & 1 & 1 & 9 & 1 & 1 & 1 & 1 & 0 & 1 & 1 & 1 & 1 & 1 \\
6 & 1 & 1 & 1 & 1 & 1 & 1 & 1 & 9 & 1 & 1 & 1 & 1 & 1 & 1 & 0 & 9 & 1 & 1 & 1 \\
17 & 1 & 1 & 1 & 1 & 1 & 1 & 9 & 1 & 1 & 1 & 1 & 1 & 1 & 1 & 9 & 0 & 1 & 1 & 1 \\
3 & 9 & 1 & 1 & 1 & 1 & 1 & 1 & 1 & 1 & 1 & 1 & 1 & 1 & 1 & 1 & 1 & 0 & 1 & 1 \\
9 & 9 & 1 & 9 & 1 & 1 & 1 & 1 & 1 & 1 & 1 & 1 & 1 & 1 & 1 & 1 & 1 & 1 & 0 & 1 \\
2 & 9 & 9 & 1 & 1 & 1 & 1 & 1 & 1 & 1 & 1 & 1 & 1 & 1 & 1 & 1 & 1 & 1 & 1 & 0
\end{tabular}

An ordinated matrix $(7 / 2) U^{Q}$.

\begin{tabular}{|c|c|}
\hline & 13712184 \\
\hline 8 & $\begin{array}{lllllllllllllllllll}0 & 1 & 1 & 1 & 1 & 1 & 1 & 1 & 1 & 1 & 1 & 1 & 1 & 1 & 1 & 1 & 1 & 1\end{array}$ \\
\hline 14 & $\begin{array}{llllllllllllllllll}1 & 0 & 1 & 1 & 1 & 1 & 1 & 1 & 1 & 1 & 1 & 1 & 1 & 1 & 1 & 1 & 1 & 1\end{array}$ \\
\hline 16 & $\begin{array}{lllllllllllllllll}1 & 1 & 0 & 1 & 1 & 1 & 1 & 1 & 1 & 1 & 1 & 1 & 1 & 1 & 1 & 1 & 1\end{array}$ \\
\hline 2 & $\begin{array}{lllllllllllllllll}1 & 1 & 1 & 0 & 1 & 1 & 1 & 1 & 1 & 1 & 1 & 1 & 1 & 1 & 1 & 1 & 1\end{array}$ \\
\hline 10 & $\begin{array}{llllllllllllllllll}1 & 1 & 1 & 1 & 0 & 1 & 1 & 1 & 1 & 1 & 1 & 1 & 1 & 1 & 1 & 1 & 1 & 9\end{array}$ \\
\hline 15 & $\begin{array}{llllllllllllllllll}1 & 1 & 1 & 1 & 1 & 0 & 1 & 1 & 1 & 1 & 1 & 1 & 1 & 1 & 1 & 1 & 9\end{array}$ \\
\hline 13 & $\begin{array}{lllllllllllllllll} & 1 & 1 & 1 & 1 & 1 & 0 & 1 & 1 & 1 & 1 & 1 & 1 & 1 & 1 & 9 & 1\end{array}$ \\
\hline 7 & $\begin{array}{lllllllllllllllll}1 & 1 & 1 & 1 & 1 & 1 & 1 & 0 & 1 & 1 & 1 & 1 & 1 & 1 & 9 & 1 & 1\end{array}$ \\
\hline 12 & $\begin{array}{lllllllllllllllll}1 & 1 & 1 & 1 & 1 & 1 & 1 & 1 & 0 & 1 & 1 & 1 & 1 & 9 & 1 & 1 & 1\end{array}$ \\
\hline 18 & $\begin{array}{lllllllllllllllll} & 1 & 1 & 1 & 1 & 1 & 1 & 1 & 1 & 0 & 1 & 1 & 9 & 1 & 1 & 1 & 1\end{array}$ \\
\hline 4 & 1 \\
\hline 5 & $\begin{array}{lllllllllllllllll}1 & 1 & 1 & 1 & 1 & 1 & 1 & 1 & 1 & 1 & 9 & 0 & 1 & 1 & 1 & 1 & 1\end{array}$ \\
\hline 11 & $\begin{array}{lllllllllllllllll}1 & 1 & 1 & 1 & 1 & 1 & 1 & 1 & 1 & 9 & 1 & 1 & 0 & 1 & 1 & 1 & 1\end{array}$ \\
\hline 19 & $\begin{array}{lllll}1 & 1 & 1 & 1 & 1\end{array}$ \\
\hline 2 & $\begin{array}{lllllllllllllllll}1 & 1 & 1 & 1 & 1 & 1 & 1 & 9 & 1 & 1 & 1 & 1 & 1 & 1 & 0 & 1 & 1\end{array}$ \\
\hline 0 & $\begin{array}{lllllllllllllllll}1 & 1 & 1 & 1 & 1 & 1 & 9 & 1 & 1 & 1 & 1 & 1 & 1 & 1 & 1 & 0 & 1\end{array}$ \\
\hline 17 & $\begin{array}{llllllllllll}1 & 1 & 1 & 1 & 1 & 9 & 1 & 1 & 1 & 1 & 1\end{array}$ \\
\hline 6 & $\begin{array}{llllllllllllllllll}1 & 1 & 1 & 1 & 9 & 1 & 1 & 1 & 1 & 1 & 1 & 1 & 1 & 1 & 1 & 1 & 9 & 0\end{array}$ \\
\hline & 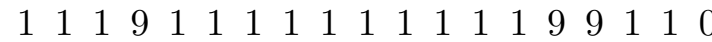 \\
\hline
\end{tabular}

Table 14

An optimally permuted matrix $(7 / 2) U^{Q^{*}}$.

This is not an isolated case. In fact, Table 15 shows an ordination of the $8 \times 35$ $\mathrm{E}\left(s^{2}\right)$-optimal design due to Cheng (1997), and provides other $8 \times p$ optimal designs as the first $p$ columns for $p=34,33, \ldots, 18$ and also as the last $p$ columns for $p=17,16, \ldots, 8$.

This design is composed of three steps:

(1) The statistical ordering provides a permutation $Q$ of columns in the original design, the columns of which are numbered in lexicographic order. 


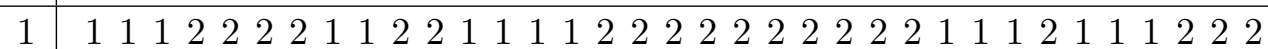

$\begin{array}{lllllllllllllllllllllllllllllllllllll}2 & 1 & 2 & 2 & 1 & 1 & 2 & 2 & 1 & 2 & 1 & 2 & 1 & 2 & 2 & 2 & 1 & 2 & 2 & 1 & 2 & 1 & 1 & 2 & 1 & 2 & 1 & 2 & 2 & 2 & 2 & 1 & 2 & 1 & 1 & 2\end{array}$

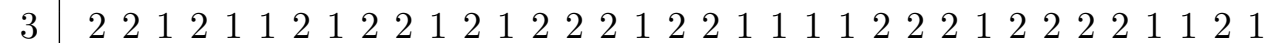

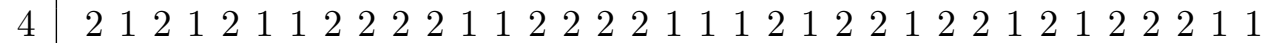

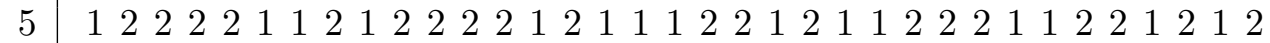

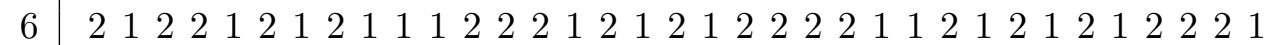

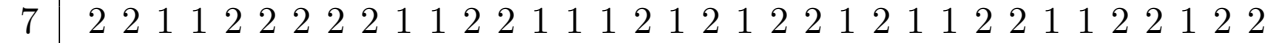

$8 \quad 1111111111111111111111111111111111111$

Table 15

An $8 \times 35 \mathrm{E}\left(s^{2}\right)$-optimal design with ordinated columns which gives an $8 \times p$ optimal design for any $p \leq 35$.

Then, Algorithm 2 with $\alpha=17$ provides the refinement $Q^{*}$ of $Q$.

(2) Looking at $U^{Q^{*}}$ reveals that

(a) Two sets $S_{1}$ and $S_{2}$ of columns comprise two separate $8 \times 7$ orthogonal designs.

(b) Among other columns, there is a set $S_{3}$ comprising the largest block of orthogonality, where $\# S_{3}=6$.

(c) The columns in $S_{1}$ should be located as the last seven columns. Moreover, it appears to be favorable to let $S_{2}$ be adjacent to $S_{1}$ and place $S_{3}$ at the start.

(3) A weighted statistical ordering on $U$ for finding "better" ordination, where the weight for each element $\chi_{i j}^{2}$ of $U$ is set as

$$
\begin{aligned}
& \left(\left\{\begin{array}{ll}
100, & \text { if } i \in S_{1} \\
1, & \text { otherwise }
\end{array}\right) \times\left(\left\{\begin{array}{ll}
100, & \text { if } j \in S_{1} \\
1, & \text { otherwise }
\end{array}\right)\right.\right. \\
& \quad \times\left(\left\{\begin{array}{ll}
0.1, & \text { if } i \in S_{2} \\
1, & \text { otherwise }
\end{array}\right) \times\left(\left\{\begin{array}{ll}
0.1, & \text { if } j \in S_{2} \\
1, & \text { otherwise }
\end{array}\right)\right.\right. \\
& \quad \times\left(\left\{\begin{array}{ll}
5, & \text { if } i \in S_{3} \\
1, & \text { otherwise }
\end{array}\right) \times\left(\left\{\begin{array}{ll}
5, & \text { if } j \in S_{3} \\
1, & \text { otherwise }
\end{array}\right)\right.\right.
\end{aligned}
$$

to locate $S_{3}, K \backslash\left(S_{1} \cup S_{2} \cup S_{3}\right), S_{2}$ and $S_{1}$ in this order, finally results in $Q^{* *}$ to furnish the design provided in Table 15.

Table 16 shows the $\mathrm{E}\left(s^{2}\right)$-value of the set of columns $\{\sigma(1), \ldots, \sigma(p)\}$ for each permutation $\sigma \in\left\{Q, Q^{*}, Q^{* *}, \bar{Q}^{* *}\right\}$, where $\bar{Q}^{* *}$ is the reverse of $Q^{* *}$.

The last example demonstrated here is the $10 \times 126 \mathrm{E}\left(s^{2}\right)$-optimal design by Cheng (1997). It is assumed that the columns are also lexicographically numbered.

Application of the statistical ordering and Algorithm 2 to the design provides the new ordination $Q^{*}$ of the columns: 


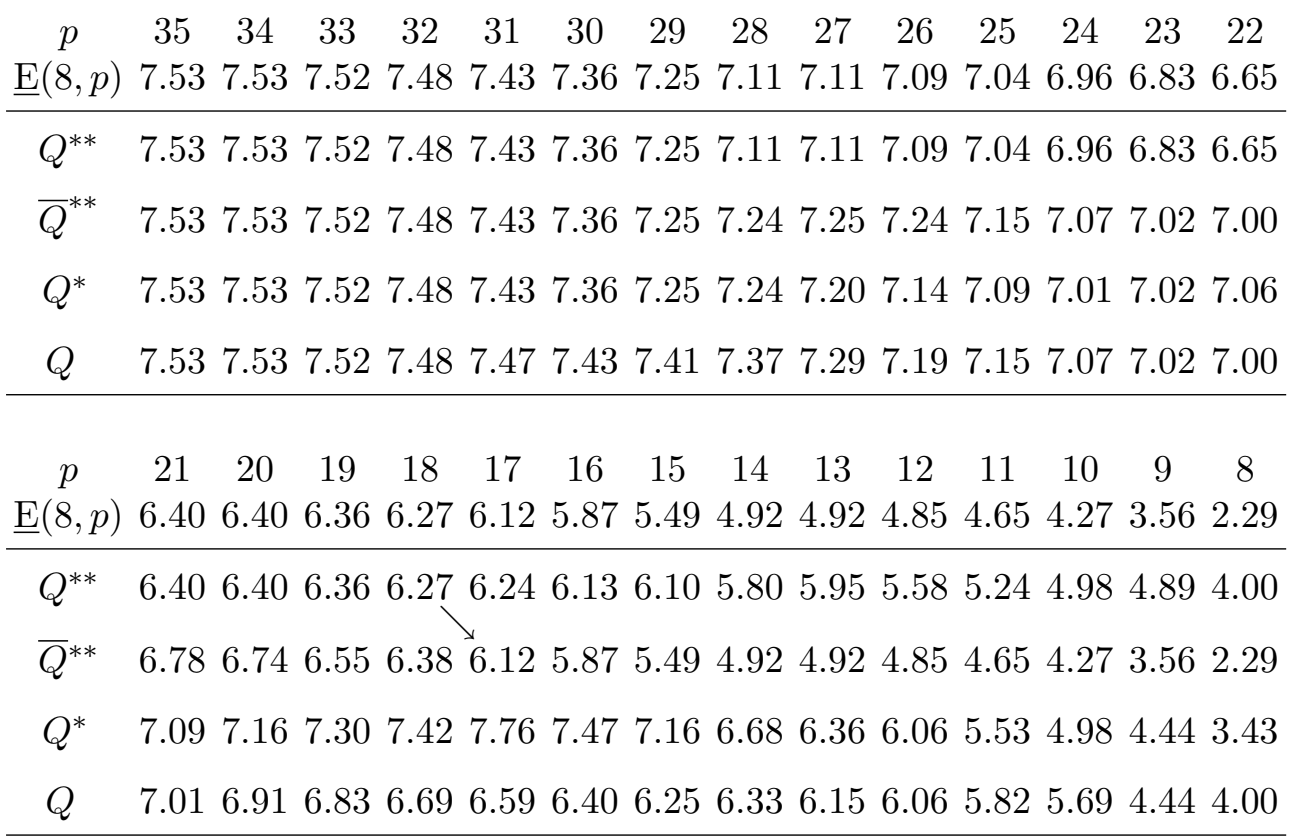

Table 16

$\mathrm{E}\left(s^{2}\right)$-values of the parts of the designs with permuted columns.

$35,27,15,116,78,16, \quad 1,39,71,104,82,126,64,97,79,34$, $100,63,21,41,122,117,59,56,33,7,42,67,95,29,24,77$, $11,85,47,114,89,55,111,96,3,84,103,115,10,101,65,58$, $28,91,40,54,14,113,9,93,86,53,72,26,49,121,102,107$, $32,119,13,69,90,6,66,74,44,92,61,51,17,25,124,76$, $2,37,108,125,18,98,45,73,81,106,36,4,22,48,62,60$, $109,87,118,43,50,94,88,12,75,20,5,23,105,52,120,112$, $68,70,38,110,46,123,8,19,57,99,83,31,80,30$.

The first $p$ columns $\left\{Q^{*}(1), \ldots, Q^{*}(p)\right\}$ of the resulting design matrix, for each $p \geq 73$, comprise a suboptimal design of which the $\mathrm{E}\left(s^{2}\right)$-value is less than $1.0025 \mathrm{E}(10, p)$. If $1.005 \mathrm{E}(10, p)$ is allowable as sub-optimality, then all the partial designs for $p \geq 49$ are suboptimal, including definitely optimal ones for $p=125,124,102,101,100,88,87,86,82,70,57,56,52$ and 51. In addition, the partial designs for $p=44$ and 28 are also $\mathrm{E}\left(s^{2}\right)$-optimal.

By applying the method discussed here to this "parent" optimal design, in an exhaustive manner, 93 of "child" $\mathrm{E}\left(s^{2}\right)$-optimal designs with 125-10 columns have been produced, excluding just 23 cases with

$123, \ldots, 115,113,108,103,95,85,77,67,59,49,41,31,23,18,13$

columns. 


\section{Acknowledgments}

This study has been partly supported by Grant-in-Aid for Scientific Research (C) 21500271, 2009, from the Ministry of Education, Culture, Sports, Science and Technology (MEXT), Japan. The authors would like to thank the editor and an anonymous reviewer who have read the manuscript carefully and provided useful comments for revision.

\section{References}

Booth, K. H. V. and Cox, D. R., 1962. Some systematic supersaturated designs. Technometrics, 4, 489-495.

Butler, N. A., Mead, R., Eskridge, K. M. and Gilmour, S. G.,2001. A general method of constructing $E\left(s^{2}\right)$-optimal supersaturated designs. J. R. Statist. Soc. B63(3), 631-632.

Bulutoglu, D. A. and Cheng, C.S., 2004. Construction of E( $\left.s^{2}\right)$-optimal supersaturated designs. Ann. Statist., 32 (4), 1662-1678.

Chen, J. and Liu, M. Q., 2008. Optimal mixed-level supersaturated design with general number of runs. Statistics 8 Probability Letters, 78 (15), 2496-2502.

Cheng, C. S., 1997. E $\left(s^{2}\right)$-optimal supersaturated designs. Statistica Sinica, 7, 929-939.

Deng, L. Y., Lin, D. K. J. and Wang, J. N., 1994. Supersaturated design using Hadamard matrix. IBM Res. Rep., RC19470, IBM Watson Research Center.

Iida, T., 1994. A construction method of two-level supersaturated design derived from L12. Jpn. J. Appl. Statist., 23(3), 147-153, (in Japanese).

Li, W. W. and Wu, C. F. J., 1997. Columnwise-pairwise algorithms with applications to the construction of supersaturated designs. Technometrics, 39(2), 171-179.

Lin, D. K. J., 1993. A new class of supersaturated designs, Technometrics, 35, $28-31$.

Lin, D. K. J., 1995. Generating systematic supersaturated designs. Technometrics, 37(2) 213-225.

Lejeune, M. A., 2003. A coordinate-columnwise exchange algorithm for the construction of supersaturated, saturated, and non-saturated experimental designs. Amer. J. Math. Manag. Sci., 23(1 \& 2), 109-142.

Nguyen, N. K., 1996. An algorithm approach to constructing supersaturated designs. Technometrics, 38(1), 69-73.

Nguyen, N. K. and Cheng, C.S., 2008. New $E\left(s^{2}\right)$-optimal supersaturated designs constructed from incomplete block designs. Technometrics, 50(1), 2631.

Nguyen, N. K. and Liu, M. Q., 2008. An algorithmic approach to constructing 
mixed-level orthogonal and near-orthogonal arrays. Computational Statistics $\&$ Data Analysis, 50(12), 5269-5276.

Nijenhuis, A. and Wilf, H., 1978. Combinatorial Algorithms, Academic Press, 2nd ed.

Plackett, R. L. and Burman, J. P., 1946. The design of optimum multifactorial experiments. Biometrika, 33, 303-325.

Satterthwaite, F.E., 1959. Random balance experimentation (with discussion). Technometrics, 1(2), 111-137.

Tang, B. and Wu, C.F. J., 1997. A method for constructing supersaturated designs and its $E\left(s^{2}\right)$ optimality. Canadian J. Statist., 25(2), 191-201.

Wu, C.F. J., 1993. Construction of supersaturated designs through partially aliased interactions. Biometrika, 80(3), 661-669.

Yamada, S., Ikebe, Y., Hashiguchi, H. and Niki, N., 1999. Construction of three-level supersaturated design. J. Statist. Plan. Infer., 81, 183-193.

Yamada, S. and Lin, D. K. J., 1997. Supersaturated designs including an orthogonal base. Canadian J. Statist., 25(2), 203-213.

Yamada, S. and Lin, D. K. J., 1999. Three-level supersaturated designs. Statist. Prob. Letters, 45, 31-39.

Yamada, S. and Lin, D. K. J., 2002. Construction of mixed-level supersaturated designs. Metrika, 56, 205-214.

Yamada, S. and Matsui, T., 2002. Optimality of mixed-level supersaturated designs. J. Statist. Plann. Infer., 104, 459-468. 